Slavica

bruxellensia

\section{Slavica bruxellensia}

Revue polyphonique de littérature, culture et histoire

slaves

$2 \mid 2009$

Femme(s)

\title{
Pour la défense de « Tarcza » de Wisława Szymborska
}

\section{Wojciech Tomasik}

Traducteur : Jeremy Lambert

\section{(2) OpenEdition}

\section{Journals}

Édition électronique

URL : http://journals.openedition.org/slavica/136

DOI : 10.4000/slavica.136

ISSN : 2034-6395

\section{Éditeur}

Université libre de Bruxelles - ULB

Édition imprimée

Pagination : 7-25

ISSN : 2031-7654

\section{Référence électronique}

Wojciech Tomasik, «Pour la défense de «Tarcza » de Wisława Szymborska », Slavica bruxellensia [En ligne], 2 | 2009, mis en ligne le 15 février 2009, consulté le 03 mai 2019. URL : http:// journals.openedition.org/slavica/136 ; DOI : 10.4000/slavica.136

Ce document a été généré automatiquement le 3 mai 2019.

\section{(c) (i) (9)}

Les contenus de Slavica bruxellensia sont mis à disposition selon les termes de la Licence Creative Commons Attribution - Pas d'Utilisation Commerciale - Pas de Modification 3.0 France. 


\title{
Pour la défense de « Tarcza » de Wisława Szymborska
}

\author{
Wojciech Tomasik
}

Traduction : Jeremy Lambert

\section{NOTE DE L'ÉDITEUR}

La langue originelle de ce texte est le polonais

1 Nous en conviendrons, la lecture des poèmes de Wisława Szymborska n'est pas toujours accompagnée de plaisir. Dans l'œuvre de la lauréate du prix Nobel de littérature de 1996, on trouve certes des chefs-d'œuvre poétiques et des ouvrages d'une grande virtuosité qui témoignent du caractère supérieur du savoir-faire littéraire de la poétesse, on trouve également des textes dans lesquels, sous la gymnastique de la langue, se laissent découvrir des strates d'idées auxquelles personne avant elle n'avait touché. On peut trouver, pour dire les choses joliment, des perles d'une valeur inestimable, qui donnent aux lecteurs un plaisir à nul autre pareil. Mais parmi les centaines de poèmes qu'elle a écrits, il s'en trouve certains au sujet desquels il est difficile de parler de plaisir et qui n'ont d'intérêt que pour l'historien de la littérature. Cette disparité qui scinde une œuvre en deux ensembles n'a rien d'exceptionnel, bien au contraire, cela reflète une chose tout à fait naturelle, qui peut s'exprimer comme suit : il faut du temps pour atteindre le niveau supérieur. La perfection artistique se paie d'années de tentatives, et parmi celles-ci seule une partie est un éclat de génie, un véritable prélude à la virtuosité. Il est de notoriété publique que la Szymborska des débuts n'est pas encore la-Szymborska-qu'on-connaît, cette certitude a été exprimée à maintes reprises, et ce, depuis longtemps. Celle-ci regagne en popularité maintenant que Szymborska a acquis le statut de «nobelisée ». On souligne souvent qu'une distance abyssale sépare cette position de ses années d'apprentissage, ce qui soulève la question de la cohérence dans sa biographie artistique. 
Cela conduit en ligne droite à la thèse du faux départ réaliste socialiste, comprise et étudiée de deux manières différentes.

2 On a tendance à considérer que Szymborska a débuté par deux fois, la première avec son recueil Dlatego żyjemy (Ce pour quoi nous vivons, 1952) et la seconde fois en 1957 lorsque parut son recueil Wołanie do Yeti (L'Appel au Yeti). Les œuvres de la maturité artistique de Szymborska créèrent une perspective à l'aune de laquelle seule sa seconde entrée sur la scène artistique peut être prise en considération. Ses premières tentatives littéraires semblent en effet être une entrée ratée dont rien, ou très peu, n'intervient dans la composition de l'image que l'on se fait de la lauréate du prix Nobel. Stanisław Balbus, excellent commentateur et exégète de la poétesse cracovienne, n'aperçoit que dans deux poèmes des années 1950, "la tonalité personnelle» que l'auteur «introduisait en fraude». Il considère le reste comme l'expression d'un engagement politique, un document de «séduction idéologique » auquel s'est adonnée une personnalité jeune et extraordinairement impressionnable ${ }^{1}$.

3 Le but que je poursuivrai ici n'est pas de prouver que tous les poèmes que Szymborska a écrit dans les années 1950 peuvent procurer du plaisir à la lecture, je n'envisage pas non plus de remuer les thèses concernant l'important retournement idéologique et artistique que connut la poétesse à l'époque du Dégel et dont témoigne Wołanie do Yeti. Je ne mettrai pas non plus en doute l'idée selon laquelle le programme réaliste socialiste a trouvé dans l'œuvre de la jeune Szymborska une réalisation exemplaire et qu'il y a donc dans l'œuvre de la «nobelisée » des poèmes condamnés à ne plus jamais être lus. Mon propos sera très limité. Je voudrais en effet démontrer que le poème «Tarcza ( (Le Bouclier) a rencontré un jugement tout à fait inapproprié. Ce n'est pas un poème majeur, il n'est en tout cas pas de ceux que l'on attend d'un auteur majeur. C'est cependant une œuvre au ton très personnel qui brise la raideur de l'expression réaliste socialiste et où apparaît, peut-être discrètement, mais en tout cas avec évidence, la-Szymborska-qu'on-connaît. Je veux défendre "Tarcza" devant l'interprétation qui fait de ce poème de la "propagande rimée » et dans laquelle apparaît le début de la route qui mena la poétesse du niveau des « raccourcis primitifs » aux hauteurs de la virtuosité littéraire. Cette tâche, je l'avoue, m'est facilitée par le fait que cette interprétation est clairement incorrecte et peu en adéquation avec la matière du poème, qu'elle en méprise le contexte historique, mais surtout qu'elle est en contradiction avec elle-même. Elle illustre en outre très clairement le fait qu'elle a été réalisée à la hâte pour être prête à un moment précis. Ce moment, c'est sans nul doute le mois d'octobre 1996, et plus précisément le moment où la poétesse reçut le prix que l'académie de Stockholm remet chaque année à un lauréat. Tadeusz Nyczek devait en effet donner à sa publication un caractère circonstanciel, afin de mettre en valeur un événement qui marquerait tout particulièrement l'histoire de la littérature polonaise. Cependant, dans la version longue de ce même livre publiée cette fois en $2005^{2}$, le chapitre consacré à « Tarcza » ne subit aucune modification.

4 La phrase suivante pourrait résumer cette désinvolture avec laquelle Nyczek lut le poème : «Tarcza » est une "élégie que Szymborska écrivit en l'honneur de la mort de Raymonde Dien » [TN 31]. L'élégie est une forme poétique très précise, dans laquelle l'accentuation des vers formant des doublons est très rigide. De plus, l'élégie est une forme littéraire qui sert une situation de souvenir douloureux, c'est une manière littéraire de parler de choses personnelles, et principalement du caractère fugace des affaires des hommes. Celui-ci est par exemple illustré par la mort d'un ami. Dans le ton de 
l'élégie, on retrouve la profonde tristesse de ceux qui sont touchés par le deuil. C'est pour cela que l'atmosphère soutenue du poème répond à celle des cérémonies funéraires.

Je laisserai de côté les problèmes formels, ils ont peu de pertinence dans la ligne interprétative que choisit Nyczek. Je me limiterai à souligner qu'à mon sens inscrire la facture textuelle de "Tarcza » dans le schéma du distique élégiaque est une entreprise périlleuse qui prouve le manque de considération pour le contenu du poème dont $a$ fait preuve le critique, qui en propose en outre de périlleuses « illustrations ». Il suffit de dire qu'on ne trouve pas dans l'œuvre de Szymborska de ces doublons caractéristiques aux accentuations diverses. J'irai même plus loin : elle n'utilise jamais la structure du distique. Elle divise son poème en cinq strophes dont l'une, celle du milieu, compte huit vers alors que les autres n'en comptent que quatre. Il n'y a que le rythme monotone du poème qui rappelle l'élégie. Ce rythme est éloquemment comparé par le chercheur à celui d'un convoi funèbre [TN 31]. Cette comparaison est liée au contenu du poème, aspect sur lequel Nyczek s'est particulièrement attardé. L'image du convoi funèbre renvoie à un groupe d'endeuillés qui accompagne le mort vers sa dernière demeure et qui participe à la cérémonie de mise au tombeau à proprement parler. Or s'il y a enterrement, c'est qu'il doit y avoir un mort. Qui est mort dans ce poème ? Szymborska commence son poème à la manière d'une enquête de personnalité, elle identifie son héroïne avec beaucoup de précision. Voici la première partie du poème (l'avant-dernière partie du poème est également la répétition de ces quatre vers) :

6 L'interprétation de Nyczek, je le répète, prend en compte ce qu'il n'y a pas dans le poème (malheureusement au prix de ce qui s'y trouve), c'est pour cette raison que dès le début, après avoir rappelé les origines de l'élégie, le critique pose la question suivante : « De quoi pouvaient être accompagnées les funérailles de Raymonde Dien?»

7 Être dans l'ignorance est une chose bien humaine. Le critique ne sait pas à quoi ressemblait l'enterrement de l'héroïne, il n'y en a pas le moindre indice dans le poème. Cette reconstruction est appelée par l'idée selon laquelle l'événement devrait, d'après les critères de vraisemblance que l'on applique à la réalité, se dérouler à la suite des événements qui sont racontés dans l'œuvre. Il serait donc intéressant de se demander ce qui est exactement raconté. Nyczek n'hésite pas un seul instant : il considère que s'il a un enterrement, c'est qu'une mort l'a précédé. L'historien se trouve face à une énigme et il s'en sort sur le mode de la spéculation, le passé lui résiste de manière inattendue, il ne lui permet pas d'établir la vérité.

8 Nyczek s'efforce de recréer la biographie de Raymonde Dien en recourrant à sa propre imagination (dans la reconstruction de l'enterrement) et à l'Histoire (en débrouillant le " problème de la mort »). On pourrait également avancer une variante plus complexe, qui voudrait que la biographie de l'héroöne du poème pouvait être, intégralement ou du moins en grande partie, rendue énigmatique, fabriquée, faite à dessein. Peut-être la presse polonaise avait-elle écrit au sujet d'une jeune communiste française, soulignant le caractère héroïque de ses actes, mais que l'héroïne en tant que telle aurait été inventée, fabriquée de toute pièce par les "propagandistes rouges acharnés ", toujours disposés à créer des mythes? Nyczek ajoute :

9 Pour interpréter « Tarcza » à sa juste valeur, il faut déterminer ce qui a servi de modèle à l'héroïne. La première question que l'on est en droit de se poser est celle-ci : l'héroïne a-telle ou non existé ? A-t-elle ou non été inventée ? Si Raymonde Dien n'a jamais existé, la réflexion tirée du caractère élégiaque du poème serait caduque. En interprétant le poème par le biais de sa forme, Nyczek devait considérer que l'héroïne de Szymborska avait une 
vie hors de la littérature. À ce stade, il était encore bien loin de découvrir qui était la jeune fille dont le portrait est proposé dans le poème, il ne pouvait pas encore comprendre à quoi cela pouvait lui servir de se retrouver dans des pages de littérature polonaise. Comment domestiquer des détails biographiques indispensables à la compréhension du sens du poème? Je vais suivre le conseil de l'historien : «Il suffit de lire des journaux et des livres. "

La fiction s'est plus d'une fois immiscée dans les biographies types proposées par la littérature réaliste socialiste. Pour mesurer la part prise par la fiction, il faut se pencher avec attention sur les arcanes de cette biographie, sur les événements et les personnages qui la composent. Les analystes des premières œuvres de Szymborska soulignent que l'auteur de Pytanie zadawane sobie (Question posée à soi-même, 1954) puisait dans les déclarations faites dans la presse, il faut donc considérer ces œuvres sur base des événements qui accompagnaient ses débuts poétiques, au début des années 1950. En d'autres termes, l'histoire des dix premières années de l'après-guerre joue un rôle considérable dans la biographie de Szymborska, cela en fait une clé d'interprétation intéressante pour chaque œuvre prise individuellement, mais également pour ses deux recueils réalistes socialistes. La grande majorité des œuvres de la période réaliste socialiste de la lauréate du prix Nobel a un caractère circonstanciel, dans ce que ce terme a de plus fort. Pour comprendre les intentions de la poétesse, il faut recréer les circonstances extérieures qui ont accompagné (ou qui ont pu accompagner) son processus créateur. Une partie de la sémantique du poème est une situation politique dans laquelle résonne la voix de Szymborska. C'est le contenu sémantique présupposé. Qu'est-ce qui constitue cette situation dans «Tarcza »? Qu'est-ce qui a attiré l'attention de la poétesse sur le personnage de cette communiste française? Que s'est-il passé pour que l'on puisse parler de cette jeune fille française sur les bords de la Vistule? Je devrai faire ce que Nyczek n'a pas fait (parce qu'il ne l'a pas voulu?) : fouiller la presse de cette époque.

11 Il serait exagéré de dire que le fait que Tadeusz Nyczek n'a pas lu la presse est un péché. Quelques années après la publication de 22x Szymborska, Wojciech Lig̨̨za s'est lui aussi mesuré à la création de la lauréate. Dans son ouvrage monographique, il s'est penché tant sur les premiers poèmes de Szymborska que sur les deux tomes de poésie écrits selon les critères réalistes socialistes ${ }^{4}$. Ceux-ci poussaient les poètes à lire consciencieusement les journaux et à écouter attentivement les commentaires portés sur leur production du début des années 1950. Une grande partie de la production poétique témoigne du fait que les auteurs lisaient les journaux, ils confirmaient que des communiqués de presse étaient à l'origine de leur poème en inscrivant, en exergue, un fragment d'information ou en indiquant que celle-ci était «tirée de la presse ». Szymborska s'est pliée plusieurs fois à ce procédé de composition, et même lorsqu'elle n'a pas estampillé son poème d'une exergue, on peut être sûr qu'à son origine préside un fait décrit dans un journal ou dans un commentaire d'une brochure de propagande. Lig̨̨za indique que « l'origine journalistique de poèmes tels que "Pieśń o zbrodniarzu wojennym", "Tarcza" et "Z Korei" est évidente " $[\mathrm{WL} 48]^{5}$ et il ajoute que » le poète réaliste socialiste n'a pas le droit d'inventer son récit » [WL 49]. Cela ne lui est pas permis car «la poésie doit compléter les journaux » [WL 48]. Szymborska s'est tenue à ces indications. "On remarque cela dans son poème au sujet de cette femme française désespérée qui se jette sous les roues d'un véhicule pour arrêter un transport d'armes à destination du Vietnam » [WL 55-56]. Le chercheur clôt sa pensée en disant que dans le poème "Tarcza", " on peut découvrir deux modèles de ballade et 
d'élégie, liés l'un à l'autre » [WL 56]. Cette note rend vraisemblable les propos de Tadeusz Nyczek, qui avait précédemment découvert des traces de l'élégie dans la construction formelle du poème.

Ne pas avoir lu la presse devient par contre un lourd péché si le lien qui unit un poème à des communiqués de presse dépasse le stade de la simple dépendance génétique. Ce qui est le cas pour « Tarcza ». Tadeusz Nyczek et Wojciech Lizęga le confirment tous les deux, mais c'est surtout le premier qui s'y attarde et qui, en tentant difficilement d'établir le sens élémentaire du poème, tombe dans l'extrapolation fantastique. Le chercheur assigne en effet, dans l'histoire racontée, un rôle pour le garçon qui, s'il ne mourut pas au Vietnam, joua de l'accordéon durant la cérémonie funéraire. Ce garçon indépendamment du fait qu'il soit, ou non, allé en Indochine, ne voulait visiblement pas de cette fille puisque celle-ci a voulu mourir sous les roues du train. Elle a donc commis un suicide. On sait qu'elle était communiste et que le train était rempli d'armes (cela ne signifie toutefois pas qu'il était blindé!), donc la jeune fille ne serait pas morte par amour, mais pour défendre ses idées, elle voulait protester contre une guerre sale. Son suicide était un " acte démonstratif de désespoir à la manière des kamikazes japonais » [WL 34]. Si elle n'avait pas été communiste, elle aurait dû passer, après sa mort, devant le jugement de Dieu. D'un point de vue pénal, Raymonde Dien a enfreint la loi, donc si elle avait survécu, elle « aurait dû être jugée en France » [WL 35]. Comment faire face à ces contradictions ? Comment savoir de qui et de quoi parle Szymborska dans son poème? En fait, il suffit de lire les journaux...

Pour l'historien de la littérature, ne pas lire est un péché très lourd. Lire de manière nonchalante également. Seule une lecture distraite et stérile pouvait mener à la conclusion que la Raymonde Dien du poème, celle dont parle « Tarcza », a mis fin à sa vie. Szymborska était passée maître dans la précision de ce qu'elle exprimait, quelques poèmes datant de l'époque où elle maitrisait réellement le réalisme socialisme viennent confirmer ce fait. Dans des fragments ou dans des phrases isolées, se rencontre cette maestria qui nous ravit quand nous savourons les poèmes qu'elle écrivit dans sa maturité artistique. Où parle-t-on de mort dans le poème? Comment Nyczek et LigĘza sont-ils arrivés à la conclusion que l'héroïne s'était suicidée sous les roues d'un train? Il est uniquement écrit que Raymonde Dien s'est «couchée sur les rails de chemin de fer ». Pour qu'elle se tue, il aurait fallut qu'elle se «jette » sur la voie. Le locuteur polonais sait que l'expression "rzucić siĘ na tory ", que l'on peut traduire par "se jeter sur une voie ", a le sens de " commettre un suicide ». Les périphrases "se jeter sous un train » ou "se jeter sous les roues" ont également ce sens. Ces trois phrases veulent dire la même chose : se tuer, s'ôter la vie. L'expression « se coucher sur les voies » n'a pas ce sens.

On retrouve le terme "położyć siĘ» ("se coucher ») dans un passage du poème qui est répété. Il y a donc deux occurrences. «Ciało » («le corps ») est quant lui mentionné une seule fois :

C'est vrai, le « corps » pourrait être "mort ». Mais rien ne propose, ni même ne suggère, une telle interprétation. Le terme « corps » est lié à « jeune fille » par au moins cent liens linguistiques. Et la majorité de ces liens renvoient à quelque chose d'agréable, de gentil, de chaud. Cette manière «vitaliste » de lire le «corps » est renforcée par l'adjectif qui apparait dans la périphrase : le corps de la jeune femme est «solide bouclier pour les jeunes filles du Vietnam ». Un bouclier est réputé « solide » s'il résiste aux coups, si on peut l'utiliser plusieurs fois et en étant sûr à chaque fois de sa solidité. Le sens de la comparaison ne serait pas suffisamment motivé si la jeune fille était morte. Raymonde 
Dien n'aurait plus pu aider personne si elle était morte, et son corps n'aurait plus pu avoir l'usage d'un bouclier. Mais dans ce poème, le mot «bouclier » («tarcza ») se trouve dans une situation particulière, il y est essentiel puisqu'il apparaît dans le titre, dont le but est d'éclairer le sens du texte. Si Raymonde Dien sert de comparaison avec ce qui est découvert durant le combat, c'est justement parce que le rôle de la jeune fille ne s'arrête pas à l'événement raconté dans le poème, et que les jeunes filles du Vietnam (et pas seulement elles) peuvent continuer à compter sur elle. Il est également important de noter que l'expression «silna tarcza " répond de façon ludique à l'expression «sexe faible », car le terme «silna » ne signifie pas uniquement « fort », mais aussi « solide ». La guerre qui était terminée (la Deuxième Guerre mondiale) et celles qui continuaient à l'époque du poème (« la sale » guerre du Vietnam, la guerre « froide ») placent une femme devant des devoirs d'hommes. C'est sur ce sujet qu'a écrit Szymborska, elle ajouta à cette idée que la source de la détermination féminine était à trouver dans un sentiment que seule une mère est capable d'exprimer.

termes « mort » et « corps » n'apparaissent qu'une seule fois dans le poème, dans une phrase qui décrit la finalité de l'activité de la jeune héroïne. Il y a, loin de la France, des villages auxquels des trains français " apportent la mort ». La " mort » est associée au lieu de la guerre, mais il est possible ici (c'est-à-dire en France) d'influer sur son déroulement. Pour ce faire, il faut agir. La jeune fille apporte un modèle de cette action. Raymonde Dien a répondu à la guerre par la guerre, et elle l'a fait, selon les termes du poème, «mue par un très grand amour ». Les coordonnées géographiques du poème sont difficiles à établir, car Szymborska utilise également le terme «ici » pour désigner le pays où «les jeunes filles, à la gare, agitent leurs mouchoirs ». C'est de ce lieu que la poétesse regarde se dérouler les choses du monde. Attardons-nous sur cette phrase :

«Je vis » a un sens double, parce qu'on peut le comprendre comme signifiant «j'habite », mais également comme "je suis vivant». De la même manière probablement pouvonsnous comprendre la construction parallèle " et elle, dans un pays... », dans laquelle le verbe a été omis. Le verbe manquant est bien sûr "vivre ", le même emploi et le même double sens sont sous-entendus : l'hérö̈ne séjourne en France, et partant, vit au moment dont il est question. On peut comprendre ce que la poétesse écrit dans « Tarcza » comme ceci : nous vivons toutes les deux, mais si elle, Raymonde Dien, habite dans un pays de malheur, moi je peux m'exprimer depuis une terre où l'on construit de nouveaux ponts et de nouvelles maisons.

«Tarcza » a paru le 7 juillet 1950 dans le périodique cracovien «ECHO TYGODNIA ». Deux jours plus tard, donc presque parallèlement à la publication du poème de Szymborska, une lettre poétique de Andrzej Braun intitulée "À Raymonde Dion" [sic] parut dans "NOWA KULTURA ». Ces deux textes avaient été précédés par un poème de Grzegorz Lasota publié dans le numéro 37 de «SZTANDAR MŁODYCH» datédu 13 juin 1950. Ce poème sans titre (dont l'incipit était Il fait étouffant dans la salle du tribunal ${ }^{6}$ ) était précédé d'une introduction tout à fait caractéristique de l'époque :

La place que réserve Lasota à cette information ne signifie pas qu'il s'agit d'une citation. C'est plutôt un digest ${ }^{8}$, un condensé d'informations trouvées dans les journaux de juin 1950, concernant une série d'événements qui s'étaient déroulés en l'espace de trois mois. Un commentaire intitulé «Pourquoi Raymonde Dien a-t-elle été condamnée? " parut dans le « SZTANDAR MŁODYCH », il résume les éléments fondamentaux de cette affaire : 
20 Le fait que Szymborska ajoute sa voix à celles qui s'élèvent pour protester contre l'emprisonnement de la jeune Française est important pour comprendre ce qu'elle a voulu dire dans "Tarcza». Ce sont toutefois les différences qui ont réellement de l'importance. En effet, d'un côté résonnent les mots des hommes en colère, et d'un autre on entend la déclaration subtile d'une femme qui a presque (je souligne ce terme) le même âge que l'héroïne. Les circonstances accentuent la différence, sur laquelle, semble$\mathrm{t}$-il, Raymonde Dien n'avait aucune influence : le lecteur polonais prend connaissance du verdict émis par le tribunal français dans les journaux du 2 juin, donc dans des numéros dont le contenu est dicté par le calendrier officiel des fêtes. En effet, il n'est pas anodin que l'histoire de la jeune communiste connaisse son plus grand retentissement à un moment proche de la Journée polonaise de l'Enfant. Cela devait être important pour les femmes, et plus encore pour les mères célibataires. La première page du numéro de "GAZETA KRAKOWSKA " qui parut le lendemain de la Journée de l'Enfant fait mention de ce titre tiré d'un communiqué de Paris : «La pacifiste Raymonde Dien a été condamnée à un an de prison par un tribunal réactionnaire français ». Ensuite, on apprend ceci :

21 La rédaction du «DZIENNIK POLSKI » publia le même texte que «GAZETA KRAKOWSKA », elle le plaça en deuxième page, avec un autre titre: «La police a chargé la foule qui s'était réunie pour manifester contre la peine de Dien $»^{11}$. Dans des numéros parus précédemment, ces deux périodiques cracoviens avaient signalé le début du procès avec des communiqués identiques («GAZETA KRAKOWSKA » lui avait donné comme titre «Le procès de Raymonde Dien commence »; «DZIENNIK POLSKI» celui-ci : «Elle a arrêté un train transportant des chars »). L'agence de presse PAP donne une description des faits en ces termes :

22 On le remarque, le tribunal français fut finalement fort indulgent, sûrement à cause de la pression du public. Le jugement qui tomba, je le rappelle, était d'un an de prison. Au printemps et en été 1950, les communistes français organisèrent une action ayant pour but la libération de la jeune fille ; une vague de protestations déferla sur tout le pays, elle toucha également la Pologne. Des écrivains polonais s'associèrent en effet aux actions destinées à libérer la communiste condamnée. Cette union de forces se révéla payante : le 24 décembre 1950, Raymonde Dien quitta les murs de la prison. En utilisant la langue de l'époque, je pourrais dire que Raymonde Dien était en 1950 une combattante à la tête du front mondial de la lutte pour la paix. La jeune Française avait, à en croire la presse, des millions d'alliés. Elle s'attendait toutefois à des poursuites. «GAZETA KRAKOWSKA » écrivit à ce sujet dans son numéro du 15 mai 1950 :

23 Le lecteur polonais n'apprenait donc rien sur les conséquences juridiques de cette action. Il ne fait pourtant aucun doute que ceux qui organisèrent ces protestations, ainsi que ceux qui y prirent part, devaient s'attendre à recevoir la même peine que celle qui attendait Raymonde Dien.

24 Si je cite de larges extraits de journaux, c'est que je veux placer «Tarcza» dans un contexte. Je suis guidé non par des détails infimes de l'histoire de la littérature, mais par la profonde conviction que la compréhension du poème de Szymborska dépend de ce que l'on a dit et écrit durant l'année 1950, et que ceci permettra d'en comprendre les destinées. C'est uniquement ainsi que sera possible une interprétation de ce poème dans sa dimension universelle, débarrassée des conditions qui dictèrent tel ou tel mot à la poétesse. J'aimerais en effet atteindre une signification qui ne serait pas contingente au temps et à l'espace, j'aimerais atteindre ce que le poème a transporté à travers les 
décennies qui nous séparent de sa production, comprendre en quoi il parle au lecteur d'aujourd'hui. Pourtant, le sens général est lié au contexte. C'est pourquoi je reviens à celui-ci et à 1950, époque durant laquelle "la poésie se battait pour la paix ». Nous trouvons cette formule entre autres dans l'en-tête d'un bloc de cinq poèmes que « POKOLENIA " publia dans son trente-quatrième numéro, daté du 3 septembre. Parmi ces œuvres, se trouvait la lettre poétique de Andrzej Braun intitulée "À Raymonde Dien ", que nous avons mentionnée plus haut, sous lequel avait été placé un dessin anonyme. Il représentait une jeune fille debout devant une locomotive à vapeur, elle avait les bras en croix, le tronc penché vers l'arrière et le torse bombé. Le dessinateur a imaginé de la sorte la situation que Braun avait tenté de dépeindre avec des mots. On se battait donc pour la paix avec des mots, mais également avec des moyens plastiques. Les premiers étaient souvent utilisés, mais les seconds n'étaient pas en reste, ce dont témoigne une toile de Andrzej Wróbelski dont le titre donnait à comprendre le sens de l'œuvre:» Raymonde Dien arrêtant un train transportant des armes vers la Corée (sic!)». Szymborska ne pouvait pas avoir vu ce tableau avant d'écrire son poème, elle ne pouvait pas non plus connaître la sculpture de Adam Smolana représentant Raymonde Dien debout, la main droite levée. La poétesse ne tirait ses connaissances que d'une seule source, qui donnait régulièrement des informations détaillées et rabâchées au sujet de la Française. Cette source, c'était la presse quotidienne qui ne manquait pas de se tenir au courant de la lutte pour la paix. Dans son numéro du 27 février 1950, le «DZIENNIK POLSKI» informait ses lecteurs comme suit dans un dossier intitulé «La lutte des ouvriers français dans la défense de la paix » :

Un mois plus tard plus ou moins, le 30 mars, «GAZETA KRAKOWSKA » mit en scène l'héroïne qui avait fait parler d'elle durant les événements de la gare de Saint-Pierre des Corps ; un passage, séparé graphiquement, de l'article intitulé « La population laborieuse de France exige la libération des patriotes emprisonnés » parlait de la jeune femme.

Nous remarquons que l'histoire de la jeune fille racontée par Szymborska comprend, dans sa version journalistique, deux points forts : le procès (extrêmement court, terminé par un jugement d'un an de privation de liberté) et la participation à la protestation, qui fut le fondement de l'acte d'accusation. Cela se passait à une époque de l'année marquée par deux fêtes très importantes pour le cérémonial communiste : la Journée de la Femme et la Journée de l'Enfant. Les premières mentions de la protestation à laquelle prit part Raymonde Dien voisinaient dans la presse avec des informations concernant la manière dont le monde développé rendait hommage à ses femmes, les informations concernant le jugement du tribunal étaient entourées d'articles consacrés aux manifestations réalisées pour la fête du premier juin. En 1950, ces deux fêtes étaient fortement liées. Parmi ces liens, celui qui me semble le plus important est celui qui découvre le mot d'ordre du rassemblement de femmes du 4 mars : «Les efforts des fauteurs de guerre se brisent sur notre volonté inflexible ». «GAZETA KRAKOWSKA » et « DZIENNIK POLSKI » couvrirent tous les deux ce rassemblement de femmes. Dans un tract, le Dr. Antonina Świerszczewska s'adressa ainsi à celles qui s'étaient réunies :

Je cite ce passage parce que j'y vois un chemin pour pénétrer dans ce petit recueil avec lequel Szymborska débuta et dans lequel parut «Tarcza ». Elle intitula ce recueil Dlatego żyjemy. Nous vivons grâce à... ces mères magnifiques, grâce au sacrifice qu'elles ont fait. L'amour maternel est un des sentiments les plus forts, de ceux qui poussent les mères à donner leur vie pour leur enfant si le danger le menace. Pour une mère, se consacrer à 
son enfant est le plus grand sacrifice. Il ne peut y avoir qu'une seule motivation à ce geste : l'amour porté aux autres enfants qui sont sauvés par ce geste.

Je ne sais pas quelles ont été les lectures de Szymborska à l'époque où elle écrivait " Tarcza », la poétesse connaissait les communiqués de presse qui attirèrent son attention sur les événements de Tours, mais également les œuvres littéraires qui devaient servir à la création artistique de la scène. Il est important de noter que la bonne foi des communiqués qui touchaient aux protestations laissait fortement à désirer. Je pense à des détails de l'histoire de Raymonde Dien qui sont rendus différemment, voire quelquefois en contradiction entre eux. Je vais dire quelques mots à ce sujet car j'ai touché une question que Tadeusz Nyczek avait déjà soulevée en notant qu'il ne fallait pas croire aveuglément la presse des années 1950. La liste des inadéquations internes (c'est-à-dire celle desquelles pouvaient se rendre compte les lecteurs des journaux et les observateurs attentifs de la vie culturelle) est longue. Les dates ne correspondent pas, le nom de l'héroïne n'est pas toujours le même, la localisation des événements est variables, etc. Pour s'approcher de ce à quoi devait ressembler l'histoire de Raymonde Dien, il faut se dégager du filtre de la presse par lequel elle fut introduite dans l'opinion publique polonaise. Je vais maintenant changer de perspective: je vais abandonner celle qui marqua le moment où fut écrit « Tarcza " pour adopter une optique actuelle.

Je commencerai par ce qui a le moins d'importance pour notre affaire, mais qu'il est toutefois bon de rappeler. Raymonde Dien vit encore actuellement, elle a presque 80 ans (elle est née en 1929) et revient volontiers sur les événements de février 1950. Dans le numéro du 27 février 1990 du quotidien «L'HUMANITÉ» (intitulé simplement «Le 23 Février 1950...»), commémorant le quarantième anniversaire de l'action de Saint-Pierre des Corps, l'héroïne se souvint avec une grande précision de ce à quoi avait pu ressembler cet événement ${ }^{18}$. En janvier 2004, elle prit part à une conférence scientifique consacrée à la « lutte contre la guerre en Indochine ». En automne de la même année, elle se rendit au Vietnam pour y célébrer le cinquantième anniversaire de la défaite française de Dien Bien Phu, pour y voir les traces de la guerre et pour y discuter avec les survivants. Le $1^{\mathrm{er}}$ septembre, en compagnie d'une délégation française, elle rencontra le secrétaire général du Parti communiste vietnamien, le camarade Nong Duc Manh ${ }^{19}$. Au terme de la conférence parisienne fut publié un recueil intitulé L'Affaire Henri Martin et la lutte contre la guerre d'Indochine. Sur les photos qui complètent l'ouvrage, on peut voir deux personnes souriantes qui furent les héros des conférences: Henri Martin y est de face et Raymonde Dien de profil, ce qui permet de remarquer que le temps n'a pas abîmé sa mince silhouette. C'est Alain Ruscio, le rédacteur du recueil, qui écrivit le texte qui répond au poème "Tarcza» et qui permet de recréer les événements de la biographie de son héroïne. Son titre est explicite : «L'Affaire Raymonde Dien $»^{20}$.

Raymonde Dien s'est couchée sur les rails devant un train qui avançait doucement sur les rails de la gare ${ }^{21}$. Elle n'était pas seule, elle était accompagnée de quelques personnes issues du groupe peu nombreux qui s'était réuni à la gare à l'appel du Parti communiste français (PCF). Les choses se passèrent sur l'aiguillage de Saint-Pierre des Corps, près de Tours, au début de l'après midi du 23 février 1950. Il s'avère que la protestation n'était pas une action spontanée, mais qu'elle avait été planifiée. Depuis un certain temps, la direction du PCF du département d'Indre-et-Loire évaluait les possibilités d'organiser une manifestation en faveur de la paix. Il est intéressant de remarquer que l'impulsion provint de quatre mères de Tours dont les enfants avaient trouvé la mort au Vietnam. La protestation dura assez peu de temps, la voie était de nouveau libre après vingt minutes. 
Le même jour, la jeune fille se rendit au commissariat de police à Tours où elle fut mise en garde à vue. Le lendemain, c'est-à-dire le 24 février, la ville fut le théâtre d'une manifestation contre l'emprisonnement de ceux qui avaient protesté sur les voies de chemin de fer. La manifestation qui suivit eut lieu le 8 mars, c'est-à-dire la Journée de la Femme. À la fin du mois de mars, Raymonde Dien fut transférée de Tours au fort Ha, à Bordeaux, où elle dut attendre son procès et le jugement du tribunal militaire français. Le procès commença le 31 mai, elle entendit la sentence, qui la privait de liberté pour un an, un peu après minuit, le $1^{\mathrm{er}}$ juin. C'était le premier procès pour « sabotage physique » de la France d'après-guerre, il se basait sur un article du code pénal que l'Assemblée nationale française avait confirmé le 8 mars, malgré l'opposition des communistes ${ }^{22}$. Dans le cas de Raymonde Dien, la procédure employée violait le principe selon lequel le droit ne peut être rétroactif. Un seul jour de la vie de la communiste française est important pour comprendre le poème de Szymborska, c'est celui dont parle "Tarcza », c'est-à-dire le 23 février 1950. Cette année-là, la jeune femme avait 21 ans. Lorsqu'elle fut arrêtée, cela faisait six mois qu'elle était mariée. Dien était son nom d'épouse. Son nom de jeune fille était Huberdeau. Le couple n'avait pas d'enfant.

31 Si je mentionne l'état civil, c'est qu'il rapproche l'héroïne de « Tarcza » d'une Szymborska un peu plus âgée. Et j'explique pourquoi la poétesse vit un fragment de son propre destin dans celui de la jeune française. Dans l'interprétation de Nyczek, un point d'interrogation apparait après le vers "Ce qu'elle fit mue par un très grand amour ». Le critique se demande ce « que signifie ce "grand amour" ? " De l'amour pour un garçon qui ne voulait pas d'elle ? Évidemment, ce n'était pas pour un garçon, mais pour... des enfants. L'amour envers les enfants est le plus grand, le plus féminin, le plus profond. Szymborska débuta après la guerre, elle avait la conviction, née à l'époque des avant-gardes, que le poète avait une mission sociale. Depuis ses débuts, elle lia l'impératif avant-gardiste de l'utilité sociale à des éléments traditionnels et difficiles à résoudre artistiquement, du moins en apparence : la versification numérique, le réalisme, le caractère épique.

Szymborska a toujours eu le souci du mot, cela marquait d'une part son économie dans ses moyens d'expression, et d'autre part son inventivité stylistique, sa vision personnelle du conceptisme. Szymborska répondit à l'abstraction des slogans idéologiques en montrant ce qu'ils signifiaient dans la vie de tous les jours, et, mieux encore, en les appliquant à des expériences très personnelles, en trouvant dans ses propres joies, dans ses propres peines, des reflets d'affaires au sujets desquelles on pouvait lire dans les premières pages des journaux du parti. La "lutte pour la paix» en est un exemple suffisamment marquant. Il ne fait aucun doute que le premier tome de Szymborska ( Dlatego żyjemy, 1952) doit son originalité au lien dont il vient d'être fait mention. La poétesse y parle sur un ton extrêmement personnel de la menace d'un nouveau conflit et du traumatisme qu'a laissé derrière elle la Deuxième Guerre mondiale : elle ne le fait pas en tant que communiste, ou en tant qu'activiste d'un mouvement pacifique, mais en tant que jeune fille, en tant que femme, et last but not least ${ }^{23}$ en tant que " mère célibataire ».

33 Lorsqu'on lit aujourd'hui les poèmes du recueil Dlatego żyjemy, il faut avoir à l'esprit la campagne agressive, liée aux mots d'ordre de défense de la paix et aux débuts du mouvement pacifiste, qui touchait la Pologne et l'Europe aux environs de 1950. C'est dans cette atmosphère de guerre froide, qui en 1950 s'était muée en guerre " chaude » en Asie, que l'on peut trouver ce qui rendait cohérent le premier recueil de poèmes de Szymborska. Dans Dlatego żyjemy, la poétesse écrit sur la nécessité de la paix en lançant des slogans dont la presse nourrissait quotidiennement ses lecteurs, elle utilise cependant 
la langue de l'intimité, des affaires privées, elle explique des problèmes de haute politique par des catégories relevant de la relation mère-enfant. Dans le recueil de poésie que Szymborska publia en 1952, nombre de ses héros sont des femmes, et une partie renvoie à l'auteur elle-même. Les œuvres réunies dans le mince volume sont des poèmes profondément personnels, dans l'indécision des sujets à traiter résonne l'écho des dilemmes vécus personnellement par l'auteur. Parmi les craintes des personnages de mères qu'elle crée dans ses poèmes, on retrouve l'inquiétude de la "mère solitaire ", selon les termes que Szymborska utilise pour se définir dans son poignant poème de son second tome de poèmes réalistes socialistes, intitulé Jako matka (En tant que mère).

La figure de la «mère solitaire " apparaît déjà dans Dlatego żyjemy et plus clairement encore dans le poème « Tarcza ». Ce poème fait partie d'un cycle de quatre miniatures qui donnèrent leur titre à l'ensemble du recueil. Il en fait partie de deux manières : d'abord en se plaçant clairement dans l'ordre de l'histoire récente, ce dont témoigne l'utilisation de noms propres authentiques et le renvoi à des événements tout aussi authentiques (la défense de Stalingrad, la protestation en France). Ensuite en construisant une image poétique sur base d'une figure paradoxale : il s'avère que si nous vivons, c'est grâce à la mort d'un étranger. Le prix de notre bonheur, dit la poétesse, a été payé par quelqu'un que nous ne connaissons même pas. De jeunes soldats périrent à Stalingrad, des fils s'en sont donc allés, laissant peut-être au monde des mères éplorées. Ce qui nous réjouit ici, sur la Vistule, a été gagné au combat là-bas, sur la Volga. Le chagrin des mères inconnues, c'est le prix qu'il fallut payer pour obtenir la paix. Et ce chagrin, c'est la femme qui le connaît le mieux. "Tarcza » est un poème sur la solidarité féminine et sur la maternité solitaire. Raymonde Dien s'est allongée sur les rails en France pour défendre de son sein, comme une mère, des enfants qui n'étaient pas les siens, des enfants vietnamiens.

Cette somme d'expériences est reflétée dans les deux ouvrages réalistes socialistes de Szymborska. Elle en avait touché un mot dans une conversation retranscrite dans « ECHO KRAKOWSKIE » peu avant la publication de Dlatego żyjemy, elle caractérisait ses centres d'intérêt par ces mots: "Le problème de la femme m'a toujours intéressé dans ses dimensions de mère et d'enfant ${ }^{24}$. Selon moi, le sens que Szymborska place dans son récit poétique sur Raymonde Dien est essentiel à la compréhension de l'ensemble de sa création, en tout cas de la majeure partie. " Tarcza » n'était pas uniquement une réaction à l'incident de l'arrêt d'un train militaire français. Parmi les ouvrages consacrés à Raymonde Dien (par exemple ceux de Lasota ou de Braun), on rencontre la forme de la lettre poétique ${ }^{25}$. «Tarcza » n'est pas une élégie (comme le suggèrent Nyczek et Lig̨̨za) ni une lettre (ce que l'on peut voir au premier coup d'œil). Cette inadéquation dans les formes littéraires montre clairement comment l'auteur traitait de manière personnelle l'histoire relatée dans les journaux.

Lorsque j'ai parlé des points essentiels de l'histoire relatée par les journaux et considérée comme cadre de " Tarcza ", j'en ai omis un volontairement. Je dois maintenant y revenir pour montrer à quoi devait servir une biographie là où elle s'établit, c'est-à-dire en France, et là où elle devint de la littérature, c'est-à-dire dans "Tarcza ». Dans le communiqué de presse du 27 février, il est fait mention de «l'arrestation de pacifistes qui, à Saint-Pierre des Corps, immobilisèrent un transport ferroviaire de chars ». La foule qui s'était réunie à Tours (" presque 8000 personnes ») exigea la libération de l'ensemble des personnes arrêtées. On sait des témoignages laissés par les participants que plusieurs dizaines de personnes s'étaient couchées sur les rails, et on connaît aujourd'hui le nom de certains d'entre eux. La presse française donna des informations sur les protestations 
menées pour s'opposer aux arrestations, et depuis la mi-mars, les communistes s'efforçaient de donner le plus grand retentissement possible à l'événement, ils tentaient de l'étendre à l'échelle du pays. Le nom de Raymonde Dien n'était alors pas encore tombé, il n'apparaît qu'à la fin du mois de mars (presque un mois après que l'affaire ait commencé). Presque immédiatement, l'opinion publique polonaise tourna son attention sur elle. Dans la presse de Cracovie, le lecteur apprenait des choses sur la jeune Française dans un contexte très particulier: "GAZETA KRAKOWSKA » et "DZIENNIK POLSKI" s'intéressaient principalement à la lettre qu'une des personnes emprisonnées à Tours avait adressée à Maurice Thorez. À la fin du mois de mars 1950, le héros collectif que constituait l'ensemble des participants à la manifestation de Saint-Pierre des Corps, fut remplacé par un seul personnage : l'expéditeur de la lettre, c'est-à-dire Raymonde Dien.

Pourquoi en fut-il ainsi ? Hilary Foolitt tenta de répondre à cette question en imaginant l'affaire Raymonde Dien sur fond de campagne de propagande communiste destinée à modifier l'image de la femme ${ }^{26}$. Cette image, ce mythe, était fortement tributaire de la Deuxième Guerre mondiale, surtout de ce que certains appelèrent déplacement (ou déblocage) de l'identité masculine. Par le biais d'événements qui sortaient de l'ordinaire, la femme (et pas seulement la femme française) put endosser deux rôles exclusivement masculins : le premier est lié à la guerre, le second au pouvoir (au gouvernement). Le mythe de la femme, né de la guerre, «humilia " les hommes. "La Nouvelle Femme » (dont on peut trouver l'origine dans la Russie bolchevique des années 1920) a une caractéristique importante, elle est détachée de ses liens sociaux antérieurs. Elle cesse de compter comme une fille, une femme, une mère, elle est maintenant combattante, communiste. Démonter cette image revient, dans un certain sens, à revenir à la tradition, à accepter le rôle de femme auquel une culture multiséculaire avait habitué les Européens. Mais le cadre de cette déconstruction est plus complexe et est lié au nom de Jozif Stalin. La fin de l'année 1949 marque le soixante-dixième anniversaire de Jozif Vissarionovič Stalin et le moment où le culte du chef fut culminant. À la tête de l'humanité progressiste, il y avait un homme, le père des nations. Le mythe de la grande famille fut ainsi créé, et Stalin était à la tête de celle-ci. Pour la femme-héroïne, c'est lié à un changement de statut: elles ne sont prises en compte que lorsqu'elles servent à l'épanouissement de la mission du chef.

La lettre à Thorez que publia Raymonde Dien était un tribut au chef. Raymonde existait par cette lettre. Si elle ne l'avait pas écrite, le monde n'aurait probablement jamais entendu parler d'elle ${ }^{27}$. Dans la culture française de l'après-guerre, le rôle des héroïnes change, ce changement est suivi d'une modification progressive du mythe, on écarte le personnage de la combattante isolée (et n'ayant pas sa place dans la société) au profit de celle qui « accompagne la vie », de celle qui, comme Jeannette Vermeersch, est proche du chef. On peut dire que les années d'après-guerre sont une période durant laquelle la femme regagna en féminité (alors qu'en même temps, on remarque le retour à un modèle patriarcal). «La Nouvelle Femme » fut incarnée de diverses façons. Il est intéressant d'en exhumer une, elle montrera clairement ce dont il s'agissait dans cette création. Ce qui constitue la nouveauté, c'est le fait que le « grand amour » féminin est effacé. La Nouvelle Femme a le droit d'aimer l'humanité et les idées, elle peut laisser de côté les particularismes sentimentaux de ses aînées. Il est vrai que Raymonde Dien doit sa signification à un homme (celui à qui elle écrit sa lettre), mais n'en conserve pas moins sa féminité. Elle cesse d'être une combattante, elle reprend son rôle de mère. C'est cela qui devait attirer Szymborska dans le personnage de Raymonde Dien. En effet, celle-ci est 
montrée dans «Tarcza » non comme celle qui aime une paix abstraite, mais comme celle qui aime ses enfants vivants. En effet, elle est un humain fait de chair et de sang, une jeune fille, une épouse. Par cet acte de création, elle normalise un tant soit peu l'univers des slogans de propagande, elle le rapproche de la qualité que cet univers refuse.

$\mathrm{Au}$ regard de l'époque à laquelle elle a été écrite, l'histoire racontée par Szymborska semble un peu surannée. Elle dit en effet qu'une grande force est placée en la femme, c'est elle qui confirme son caractère maternel. C'est dans le mouvement de protection de son propre enfant face au mal du monde, qu'il faut chercher les principes d'une idéologie et les mots d'ordre du combat pour la paix, de la guerre contre la guerre. L'idéologie ne dicte pas une manière de vivre. Au contraire, ce sont les choix idéologiques qui guident la vie. S'esquisse ici une perspective par laquelle on peut imaginer la jeunesse de l'auteur de Wołanie do Yeti: la jeune Szymborska accepta le communisme car il entrait en convergence avec les valeurs qui étaient proches de la poétesse. Ce choix n'était nullement dicté par la conjoncture, il répondait à une croyance de jeunesse selon laquelle il est possible de s'opposer au mal et que les communistes connaissent les moyens de parvenir à cette fin. Je pourrais également utiliser une autre perspective pour comprendre cette idée. Le changement de ligne interprétative de la propagande communiste (liée au culte de Stalin et à la réinterprétation du mythe de la femme) convainquit Szymborska qu'elle suivait la voix de ceux qui en 1950 avaient fondé un mouvement pacifiste qui comptait plusieurs millions de membres.

"L'HUMANité », dans son numéro du 2 juin 1950, dit de Raymonde Dien, qui vient juste d'être condamnée, qu'elle était " presque une enfant $»^{28}$. Szymborska dit de son hérö̈ne qu'elle « avait l'âge de nos plus jeunes ouvriers ». Destinée, âge et sexe sont tous inscrits dans le personnage de l'héroïne. Elle est une «mère célibataire » qui se bat pour ses enfants vietnamiens, mais également une enfant en qui se reflète tout le monde progressiste. Dans son geste poétique, elle répète ce dont parle le poème. Dans l'un comme dans l'autre nous rencontrons un bouclier (tarcza). Une jeune femme française défend des enfants vietnamiens, et elle est mise en scène par une poétesse à peine plus âgée qu'elle. Ce poème qui a pour sujet un bouclier est lui-même une œuvre-bouclier. Les protestations de l'opinion publique hâtèrent le terme de la guerre d'Indochine, ce qui économisa la vie de milliers d'enfants, et Raymonde Dien fut libérée. Le geste de la jeune fille figure l'image d'un bouclier, le poème également.

41 Après son poème sur Raymonde Dien, Szymborska utilisa une fois encore une scène dans laquelle quelqu'un se couche sur des rails de chemin de fer. Cette fois, l'héroïne sera une ombre, celle d'une reine, qui « se couche sur la voie $»^{29}$. Ce récit au sujet d'une ombrebouffon pourrait être le sujet d'un article. Dans celui-ci, je défends « Tarcza », car je pense que ce poème le mérite. On y rencontre en effet la-Szymborska-qu'on-connaît.

\section{NOTES}

1. Voir Balbus St., Świat ze wszystkich stron świata. O Wisławie Szymborskiej. Aneks: Wisława Szymborska. Dwadzieścia jeden wierszy (Le Monde de tous les côtés du monde. Au sujet de Wisława 
Szymborska. Annexe: Wisława Szymborska. Vingt et un poèmes),Wydawnictwo Literackie, Cracovie, 1996, pp. 16-17. Andrzej Zieniewicz a très bien formulé la thèse du double départ : voir notamment Alergia na słuszność (Poezja Wisławy Szymborskiej w świetle jej debiutu) (Allergie à la légitimité [La Poésie de Wisława Szymborska à la lumière de ses débuts littéraires]), in : « ROczNIK TOWARZYSTWA LITERACKIEGO IM. ADAMA MICKIEWICZA», 1996, pp. 65-85. Le chercheur écrit ceci à propos de la première plaquette de poésies de la poétesse : "Szymborska produit de beaux poèmes irréels en acceptant leur affectivité démonstrative.» (p.69) Les véritables débuts devaient venir lorsque l'auteur "passa d'une conception "respectable" de la poésie comme manifestation d'une communauté de sentiments, à une conception "moniste" de la poésie " (p. 74). Un regard général sur l'ensemble de l'œuvre de Szymborska ressemble donc à ceci : "Toute la création de Wisława Szymborska est "à concevoir" comme une réaction allergique à ses débuts socio-littéraires "légitimes" durant les années 1950. » (p 76) D’autres se sont exprimés sur la beauté dans la biographie créatrice de Szymborska, notamment Artur Sandauer: Voir Na przykład Szymborska. („Samobójstwo Mitrydatesa” ciąg dalszy. Egzemplifikacja) (Par exemple Szymborska. [«Le Suicide de Mithridate ». Exemplification]), in : « MIESIĘCZNIK LITERACKI » $n^{\circ} 4$, 1968. Voir également Matuszewski R. : Wisława Szymborska. Portret pisarzy trzydziestolecia (Wisława Szymborska. Portrait des écrivains trentenaires), in : « LITERATURA », 1974, nº 25.

2. Voir Nyczek T., 22x Szymborska, a5, Poznań, 1997 et Tyle naraz świata. 27x Szymborska, a5, Cracovie, 2005. Les extraits cités sont issus de cette édition, notée par les lettres TN suivies du numéro de page correspondant.

3. Szymborska W., « Tarcza », in : Dlatego Żyjemy, Wydawnictwo Literackie, Cracovie, 1952. Tous les extraits de poèmes de Szymborska cités dans cet article ont été traduits par Jeremy Lambert.

4. Lig̨̨za W., o poezji Szymborskiej. Świat w stanie korekty (Au sujet de la poésie de Szymborska. Le monde dans un état de correction), Wydawnictwo Literackie, Cracovie, 1999. Les extraits cités sont issus de cette édition, notée par les lettres WL suivies du numéro de page correspondant.

5. Les trois poèmes cités pourraient être traduits respectivement par : "Chant sur un criminel de guerre », « Le Bouclier » et « De Corée » $(\mathrm{NdT})$.

6. «W Sali sĄdowej jest duszno ».

7. Lasota Grz., Sans titre (inc. : «W Sali sĄdowej jest duszno » [ « Il fait étouffant dans la salle du tribunal »]), in : «SZTANDAR MŁODYCH », 1950, $\mathrm{n}^{\circ} 37$, p. 2.

8. En anglais dans le texte (NdT).

9. «Dlaczego Raymonde Dien została skazana » (« Pourquoi Raymonde Dien a été condamnée »), in : «SZTANDAR MŁODYCH », 1950, n 30, p. 6.

10. «Bojowniczka pokoju Raymonde Dien skazana na rok wį̨zienia przez reakcyjny sąd francuski » («La pacifiste Raymonde Dien a été condamnée à un an de prison par un tribunal réactionnaire français »), in : «GAZETA KRAKOWSKA », 1950, $\mathrm{n}^{\circ} 150, \mathrm{p} .1$.

11. «Policja szarżowała na tłum demonstrujĄcy przeciwko wyrokowi na Dien » (« La police a chargé la foule qui s'était réunie pour manifester contre la peine de Dien »), in : «DZIENNIK POLSKI » , 2 juin 1950, p. 2.

12. «Proces Raymonde Dien rozpoczĄł siĘ» (« le procès de Raymonde Dien commence »), in : «GAZETA KRAKOWSKA », 1950, n 148, p. 1 ; «Zatrzymała pociĄg z czołgami » (« Elle a arrêté un train transportant des chars »), in : «DZIENNIK POLSKI», 31 mai 1950, p. 2.

13. « Z światowego frontu bojowników o pokój» («Du front mondial des combattants pour la paix »), in : «GAZETA KRAKOWSKA », 1950, n 133, p. 2.

14. «Walka robotników francuskich w obronie pokoju» («La lutte pour la paix des ouvriers français »), in : «DZIENNIK POLSKI », 27 février 1950, p. 1.

15. «Ludność pracujĄca Francji domaga się uwolnienia wiĘzionych patriotów » («Le peuple laborieux de France exige la libération des patriotes emprisonnés »), in : «GAZETA KRAKOWSKA », $1950, n^{\circ} 89$, p. 2. 
16. L'auteur cite ce texte dans la forme sous laquelle il est imprimé dans «GAZETA KRAKOWSKA ». 17. «O naszĄ niezłomnĄ wolę rozbijajĄ sį̨ wysilki podżegaczy wojennych. Wiec kobiet krakowskich z okazji MiĘdzynarodowego Dnia Kobiet » («Les efforts des fauteurs de guerre se brisent sur notre volonté inflexible. Rassemblement de femmes cracoviennes à l'occasion de la Journée Internationale de la Femme »), in : «GAZETA KRAKOWSKA », 1950, n 64, p. 1.

18. «Le 23 février 1950... », in : «L'HUMANITÉ », 27 février 1990, p. 2.

19. «Party leader hails French friends visiting Vietnam» («Le chef du parti salue les amis français qui visitent le Vietnam »), in : «VIETNAM NEWS », $1^{\text {er }}$ septembre 2004, p. 1 ; «French peace activists who rallied to Uncle Ho's cause» («Ces pacifistes français qui ont rallié la cause de l'Oncle Ho »), in : « VIETNAM NEWS », 6 septembre 2004, p. 1.

20. Voir Ruscio A., "L'Affaire Raymonde Dien », in : L'Affaire Henri Martin et la lutte contre la guerre d'Indochine, Ruscio A. (dir.), Le Temps des Cerises, Pantin, 2005. L'auteur de cet article se sert de ce texte dans sa reconstruction des événements de « l'affaire » Raymonde Dien.

21. Les wagons qui transportaient le matériel de guerre étaient tirés par une locomotive électrique. Les reconstructions plastiques de cet événement en Pologne saluent la tradition du $\mathrm{XIX}^{\mathrm{e}}$ siècle, elles montrent Raymonde Dien barrant le passage à une locomotive à vapeur.

22. Des articles informèrent le lecteur polonais des modifications mentionnées. Voir notamment l'article «Reakcyjna wiĘkszość francuskiego Zgrom[adzenia] Narodowego uchwaliła ustawĘ represyjnĄ» («La majorité réactionnaire de l'Ass[emblée] nationale françaisea voté un règlement répressif »), in : «GAZETA KRAKOWSKA », 1950, n 68, p. 2.

23. En anglais dans le texte (NdT).

24. Płatowicz B., «Rozmowy z pisarzami » («Conversations avec des écrivains »), in : «ECHO KRAKOWSKIE », 1951, n 103, p. 4.

25. La forme de la lettre poétique fut également utilisée par d'autres poètes qui s'étaient documenté sur la vie de la Française (Voir Jaworski K. A., «Do Raymonde Dien » [ À Raymonde Dien »], in : « TYDZIEŃ LITERACKI » [Supplément au «SZTANDAR LUDU »], 1950, n 4, p. 2). Parmi ceuxci, certains publièrent leur poème au moment où l'affaire trouvait une conclusion et que la jeune femme fut libérée (Voir Słobotnik W., «Do Raymonde Dien» [«À Raymonde Dien»], in: «PANORAMA » [Supplément au «DZIENNIK ŁóDZKI»], 1952, n 1, p. 1, ou Safrin H., " Do Raymonde Dien » [«À Raymonde Dien »], in : «EXPRESS ILUSTROWANY», 1953, n 76, p. 3). L'analyse de ces expressions poétiques mériterait une étude particulière, elle devrait prendre en compte les traductions des œuvres françaises auxquelles le lecteur polonais pouvait avoir accès (Voir Depestre R., « Kartka do Raymonde Dien » [« Une page pour Raymonde Dien », trad. en polonais par J. Lisowski, in : «NOWA KULTURA », 1952, n 51/52, p. 8, ou Riffaud M., Idem). Le poème le plus important concernant Raymonde Dien (Guillevic E., "Raymonde Dien», in: «L'HuMANITÉ», 4 novembre 1950, p. 2) ne fut pas traduit en polonais. Il faut également ajouter que Henri Martin, le second jeune héros de la «lutte pour la paix » qui fut, lui, condamné à cinq ans de prison, ne trouva pas en Pologne un soutien comparable à la campagne littéraire consacrée à la défense de Raymonde Dien. En 1950, les murs des villes françaises étaient couverts d'affiches appelant à la libération tant de Raymonde Dien (ce qui apporta des résultats en décembre) que de Henri Martin (ce qui ne changea en rien la situation du jeune communiste, qui passa plus de trois ans en prison). Selon les historiens, il s'agissait de deux actions menées en parallèle par les communistes. En 1950, Krzysztof Gruszczyński publia PociĄg do Marsylii, une pièce qui racontait le sabotage d'un train transportant des armes. Le machiniste, qui est un des protagonistes principaux, s'appelle André Martin. Je n'exclus pas qu'il s'agisse d'un hasard. Dans cette histoire, comme dans d'autres œuvres reflétant des faits de cette époque, on trouve des éléments tirés d'histoires différentes : dans ce cas, l'affaire se passe dans « une petite ville du sud de la France » (Voir Pociąg do Marsylii. Sztuka $w$ trzech aktach [Le train de Marseille. Pièce en trois actes], 
Czytelnik, Varsovie, 1952, p.5). On peut y voir clairement un écho des évènements de SaintPierre des Corps.

26. Foolitt H., "Woman and (Cold) War. The Cold War creation of the myth of "La Française résistante" » (La femme et la Guerre [Froide]. Le mythe de la " Française résistante » créé par la Guerre Froide), in : « FRENCH CULTURAL STUDIES », 1997, vol. 8., pp. 41-51.

27. Le 28 avril 1950, Maurice Thorez fêta son cinquantième anniversaire. À cette occasion, le secrétaire général du PCF reçut de nombreux cadeaux, des souvenirs, des dépêches de félicitation et des mots de circonstance. La lettre que Raymonde Dien lui écrivit de prison, et dans laquelle elle s'engageait à poursuivre son combat pour la paix, entre dans le contexte de cet anniversaire. En Pologne, le jubilé de Thorez fut entre autres marqué par la publication de son autobiographie intitulée Syn Ludu (Le Fils du peuple, 1937).

28. Durand P., « Raymonde Dien condamnée à un an de prison », in : «L'HUMANITÉ », 2 juin 1952, p. 2.

29. 29 Szymborska W., «Cień » (L’Ombre), in : Sól (Le Sel), Państwowy Instytut Wydawniczy, Varsovie, 1962.

INDEX

Index géographique: Pologne

Index chronologique : communisme, XXe siècle

Mots-clés : littérature polonaise, réalisme socialiste

oeuvretraite Szymborska W. : « Tarcza » (in Dlaczego żyjemy)

\section{AUTEURS}

WOJCIECH TOMASIK

Professeur à l'Université de Bydgoszcz 